\title{
Single-dose Levodopa Administration and Aging Independently Disrupt Time Production
}

\author{
Brian C. Rakitin ${ }^{1}$, Nikolaos Scarmeas ${ }^{1}$, Tina $\mathbf{L i}^{1}$, Chariklia Malapani, ${ }^{1,2}$, \\ and Yaakov Stern ${ }^{1}$
}

\begin{abstract}
We tested the hypothesis that age-related time production deficits are dopamine-mediated. The experiment was conducted double-blind, and with random assignment of 32 healthy aged and 32 healthy young participants to either inert placebo or levodopa (200 $\mathrm{mg}$ ) groups. The procedure included training participants to produce two target time intervals ( 6 and $17 \mathrm{sec}$ ) in separate blocks, drug/placebo administration, a 1-hr delay, and then delayed free-recall time production retesting without feedback. Participants also performed a speeded choice reaction time (RT) task, as a control for potential dopaminergic and aging effects on attention and psychomotor speed. Results
\end{abstract}

\section{INTRODUCTION}

There is an increasingly broad literature demonstrating that aging is associated with impaired interval timing abilities. These experiments have taken place during a period of growing empirical and theoretical understanding of the neuroanatomical, pharmacological, and cognitive underpinnings of interval timing (Ivry \& Spencer, 2004; Gibbon, Malapani, Dale, \& Gallistel, 1997). Among these findings are demonstrations of specific dopamine (DA)-dependent timing impairments in Parkinson's disease (PD) patients, as well as animal studies indicating the involvement of the dopaminergic systems. Also relevant are studies indicating that age-related impairment to (nontemporal) memory and attention stem at least in part from loss of DA-dependent frontal function (Braver \& Barch, 2002). These facts, combined with certain similarities between the age and PD-related timing impairments (Rakitin, Stern, \& Malapani, 2005; Malapani, Rakitin, Fairhurst, \& Gibbon, 2002), motivated the current study examining the effects of a DA-based pharmacological intervention on age-related time production impairment.

Time production refers to the act of matching response latency to the duration of a remembered target interval in discrete trials. ${ }^{1}$ Time production by healthy young subjects has two characteristic psychophysical

${ }^{1}$ Columbia University, ${ }^{2}$ New York State Psychiatric Institute indicate that during retesting, aged participants show durationdependent timing errors that are larger than those shown by the young participants. Levodopa administration yielded lengthened time production of both target intervals. The aging and levodopa effects did not interact. Also, aging slowed RT and increased RT variability, but levodopa had no effect on the RT. These results suggest that at this dosage and under these specific conditions, timing is dopamine-mediated but the effect of aging on time production is not. Moreover, the levodopa timing effect cannot be attributed to the effects of dopaminergic function on psychomotor speed. qualities, accuracy and scalar variability (Rakitin, 2005; Rakitin, Gibbon, et al., 1998; Ivry \& Hazeltine, 1995; Wearden \& McShane, 1988). Accuracy refers to the absence of systematic deviations in average response latency from the target intervals. Scalar variability refers to the tendency for intraindividual response latency variability measured by the standard deviation to increase in proportion to the average response latency. The standard information processing model of this and other timing behaviors includes a time basis ${ }^{2}$ (i.e., the apparatus for the subjective representation of currently elapsing intervals), memory, and decision stages (Zakay \& Block, 1997; Gibbon, Church, \& Meck, 1984; Gibbon \& Church, 1983; Treisman, 1963), collectively referred to as the "internal clock." The time basis consists of a variable speed pacemaker, a switch, and an accumulator. The accumulator serves as a "sensory store" or "working memory" for time, the activity of which increments with real time if attention is directed toward time, closing the switch, and allowing pacemaker pulses to increment the accumulator. Intervals marked as relevant by feedback or reinforcement enter into "reference" memory, for comparison to the current accumulator value and the decision as to whether or how to respond.

Although timing relies on the functioning of many neurochemical systems (Meck, 1996), animal studies have implicated dopaminergic neurotransmitter systems in the control of two aspects of the time basis, the pacemaker and the switch. Indirect DA agonists such as 
methamphetamine (Maricq \& Church, 1983; Meck, 1983; Maricq, Roberts, \& Church, 1981) speed up the pacemaker, whereas DA antagonists such as haloperidol (Meck, 1983, 1986; Maricq \& Church, 1983) slow down the pacemaker. These same drugs have contrasting effects on animals' ability to selectively attend to timing stimuli (Buhusi \& Meck, 2002; Santi, Weise, \& Kuiper, 1995). Specific $\mathrm{D}_{2}$ receptor agonists such as quinpirole only affect attention to time (Stanford \& Santi, 1998; Frederick \& Allen, 1996). Whether a particular DA agent affects both pacemaker speed and attention may depend on whether the drug has affinity for the $\mathrm{D}_{1} \mathrm{DA}$ receptor in addition to the $\mathrm{D}_{2}$ receptor (Stanford \& Santi, 1998; Frederick \& Allen, 1996; Meck, 1986).

Pharmacological studies of timing with healthy young human participants are fewer in number and primarily use temporal discrimination tasks (i.e., judging a stimulus as longer or shorter in duration than a standard interval). These studies generally support the models derived from the animal experiments. For example, haloperidol reduces the precision of temporal judgments, an effect consistent with reduced pacemaker speed (Rammsayer, 1989, 1994, 1997, 1999). Drugs which act to either increase (e.g., levodopa) or decrease (e.g., AMPT) DA in the striatum had no effect on short interval discrimination (Rammsayer \& Vogel, 1992; Rammsayer, 1989), suggesting that DA receptor activity rather than DA levels were responsible for the timing effects. These studies also suggested that discrimination and estimation of longer $(>0.5 \mathrm{sec}$ ) intervals is sensitive to drugs (e.g., remoxipride) that primarily affect the mesolimbocortical DA, whereas very brief interval discrimination is selectively impaired by haloperidol, which affects the mesostriatal DA system (Rammsayer, 1994, 1997). Evidence that benzodiazepine and cholinergic agonists that affect memory function also affect timing of these longer intervals suggests a role for DA in the modulation of temporal memory (Rammsayer, 1997, 1999).

Parkinson's disease provides an opportunity for studying the effects DA has on timing because it produces DA depletion in the striatum (Kaasinen \& Rinne, 2002; Agid et al., 1989; Hornykiewicz, 1989). Most convincing are those studies that have directly linked DA to deficits in time discrimination (Pastor, Artieda, Jahanshahi, \& Obeso, 1992), time production (Malapani, Deweer, \& Gibbon, 2002; Malapani et al., 1998; Pastor, Artieda, et al., 1992), and repetitive tapping (O'Boyle, Freeman, \& Cody, 1996; Pastor, Jahanshahi, Artieda, \& Obeso, 1992; cf. Diedrichsen, Ivry, \& Pressing, 2003 for an alternative view) by comparing PD patients on and off DA replacement therapy (i.e., levodopa). In some cases, DA-mediated timing errors are consistent with a slowed pacemaker (e.g., Pastor, Artieda, et al., 1992). However, Malapani and Rakitin (2003) and Malapani, Deweer, et al. (2002) demonstrated DA-related timing deficits related to temporal memory encoding and retrieval. The PD results are congruent with the pharmacology studies to the extent that they implicate both pacemaker and temporal memory systems. They differ in that the use of levodopa as the active agent implicates striatal DA levels in the control of temporal behavior.

In lines of research unrelated to the DA hypothesis, many studies report that aging adversely affects timing. A meta-analysis of studies of timing and aging (Block, Zakay, \& Hancock, 1998) indicated that verbal estimations grew shorter with age, verbal productions grew longer with age, and temporal production was unaffected by age. These effects were attributed to age-related decrease in attention to time and the corresponding effect on the switch. More recent reports confirm the general finding of attention-mediated timing problems in aging (Lustig \& Meck 2001; Craik \& Hay, 1999; Vanneste \& Pouthas, 1999), and also report age-related deficits in time production (Rakitin, Stern, et al., 2005; Perbal, Droit-Volet, Insingrini, \& Pouthas, 2002; Craik \& Hay, 1999). Degraded temporal memory in aging has also been inferred. Time reproductions under distraction shortened with age, and were associated with poorer working memory performance (Perbal et al., 2002). Other studies have found progressively noisy encoding of temporal values with increasing age (McCormack, Brown, Maylor, Darby, \& Green, 1999; Wearden, Wearden, \& Rabbitt, 1997), and time production errors restricted to delayed free recall (Rakitin, Stern, et al., 2005; Malapani, Rakitin, et al., 2002).

No studies exist directly linking DA function to agerelated timing deficits. However, there is ample evidence that DA function declines with age. Postmortem studies have demonstrated that DA in the striatum decreases with age (e.g., Haycock et al., 2003) at approximately $40 \%$ of the rate found in PD (Agid et al., 1989), and with a more even distribution across the striatum (Hornykiewicz, 1989). PET imaging of the DA system indicates normal age-related loss of $\mathrm{D}_{1}$ (Suhara et al., 1991) and $D_{2}$ (Volkow et al., 1996) receptors across the brain, but especially in the striatum and frontal cortex (Kaasinen \& Rinne, 2002; Kaasinen et al., 2000; Suhara et al., 1991). PET imaging studies have also linked age reductions in $\mathrm{D}_{2}$ to decreased executive and motor function (Kaasinen \& Rinne, 2002; Backman et al., 2000; Volkow et al., 1998). Age-related deficits in cognitive function have been treated with DA agonists (Arnsten, Cai, Steere, \& Goldman-Rakic, 1995; Ollat, 1992), supporting the functional imaging findings.

The above review establishes several facts: (1) timing relies on DA, (2) DA declines in aging, and (3) timing in aging is abnormal. From these facts, the question arises as to whether age-related timing problems stem from loss of dopaminergic function.

In order to test this hypothesis, we chose to examine the effects of levodopa on delayed free-recall peakinterval (PI) time production. The motivation for the choice of procedure is the apparent similarity in impairments observed in both PD (Malapani, Deweer, et al., 
2002) and healthy aged (Rakitin, Stern, et al., 2005) participants in delayed free-recall PI performance, suggesting a shared information processing basis (Rakitin, Stern, et al., 2005; Malapani \& Rakitin, 2003). We theorize that the duration-dependent errors shown by both groups-the tendency to overproduce the shorter of two intervals while underproducing the longer interval to a greater (as in PD) or lesser (as in healthy aging) extent-stem from alterations to the accumulator (i.e., working memory). These alterations cause the accumulator value to increase slowly for short intervals but to accelerate as intervals elapse (Shea-Brown, Rinzel, Rakitin, \& Malapani, in press; Malapani \& Rakitin, 2003). As a result, subjective time lags behind real time for short intervals and leads real time at longer values producing the characteristic duration-dependent timing errors. Regardless of the proposed cognitive mechanism (for an alternative proposal, see Meck \& Benson, 2002; Mattle \& Meck, 2000), further similarity between the PD and aging effects can be established by demonstrating that the aging effect is DA dependent.

Another advantage of the delayed free-recall PI procedure is its complexity. The requirement that participants retain and then produce two target seconds-long intervals without the advantage of feedback adds temporal memory and attention requirements that may make the task more sensitive to DA function than the time perception task previously shown to be insensitive to levodopa (Rammsayer, 1989).

The choice of levodopa as the DA agent in this experiment reflects the fact that levodopa fully reverses the PD impairment (Malapani, Deweer, et al., 2002) and medicated PD patients performed better than their agematched controls, potentially indicating a reversal of a baseline aging effect (Malapani, Rakitin, et al., 2002). The 200-mg dose of levodopa was chosen to exceed the dose that previously failed to alter time perception thresholds (Rammsayer, 1989), and to generate tolerable side effects in both young (Hasbroucq, Tandonnet, Micallef-Roll, Blin, \& Possamaï, 2003; Micallef-Roll, Rihet, Hasbroucq, Possamai, \& Blin, 2001) and old (Newman, LeWitt, Jaffe, Calne, \& Larsen, 1985) participants. Our hypothesis is that administration of levodopa to participants during the retention interval will attenuate the durationdependent timing errors observed in aged individuals. This hypothesis depends on the assumption that the aging deficit stems from reduced DA levels, which would be increased by levodopa (Misu, Goshima, Ueda, \& Okamura, 1996). In contrast, the increased DA levels produced by levodopa would have little effect if the deficit was related instead to reduced receptor density (e.g., Kaasinen \& Rinne, 2002).

Levodopa also produces a measurable decrease in reaction time (RT: Hasbroucq et al., 2003; Micallef-Roll et al., 2001). These findings regarding the effects of levodopa on psychomotor speed motivated us to include a choice RT task as a control. That is, if levodopa affects both RT and time production in the same direction, the effects would be attributable to changes in psychomotor speed, rather than to the specific effects of levodopa on time production.

\section{RESULTS \\ Side Effects}

Of the 16 elderly subjects who took levodopa, 7 (44\%) reported mild nausea. Of these, 3 (43\%) experienced it only during the break before testing, and 4 (57\%) experienced it during testing. Of the 16 young subjects who took levodopa, 7 (44\%) reported mild nausea. Of these, 4 (57\%) experienced it only during the break before testing, and 3 (43\%) experienced it during testing. In addition, one young control participant reported nausea during testing due to an unrelated stomach ailment.

\section{Time Production}

Individual participants' (symbols) and group mean (lines) time production accuracy (i.e., $M D$ ) data are presented in Figure 1. For all four groups and both target intervals, the solid lines represent the group mean baseline production performance, which was very close to the target intervals' duration, indicating accurate baseline time production, on average. The main effect of duration was significant $[F(1,60)=2260.58, p<.0001]$. There was also a significant Session $\times$ Duration interaction $[F(1,60)=1956.53, p<.0001]$, reflecting the fact that during testing all four groups overproduced the short interval, and three of the four groups underproduced the long interval, relative to baseline.

Comparing the dashed lines in the two right panels to the dashed lines in the two left panels, one can see that group mean time productions during the retesting session are longer following administration of levodopa compared to placebo. The size of this levodopa-induced overproduction is $0.87 \mathrm{sec}$, averaged over target intervals and age groups, and subtracting baseline performance. This effect corresponds to a significant Session $\times$ Drug group interaction $[F(1,60)=4.49, p<.05]$. The main effect of drug group was also significant $[F(1,60)=4.86$, $p<.05$ ]. Also evident in Figure 1 is the relatively even distribution of values associated with individuals with nausea during the retesting session. Excluding these individuals from the analysis increased the magnitude of the levodopa effect to $1.05 \mathrm{sec}$, and significance of both the Session $\times$ Drug group interaction $[F(1,52)=$ $5.12, p<.05]$ and the main effect of drug group $[F(1,52)=6.03, p<.05]$. In the analysis that subdivided the two levodopa groups into those who did and did not experience nausea at any point during the experiment, the only significant effect was that of session $[F(1,28)=$ $4.60, p<.05]$. It is therefore unlikely that the levodopa 
Figure 1. Time production $M D$ data. The four panels each contain data for all 16 participants in one of the four groups, plus the group means. The four symbols in each column of each panel indicate one participant's mean $M D$ s for the 6-sec baseline (lower circles), the 17-sec baseline (upper circles), 6-sec testing (lower triangles), and 17 -sec retesting (upper triangles) sessions. Participants that experienced nausea during the retesting sessions are denoted with small symbols. Within each panel, the participants are sorted from left to right in order of increasing 17 -sec retesting session $M D$. The solid lines are the group means for the baseline sessions and the dashed lines are the group means for the retesting sessions.
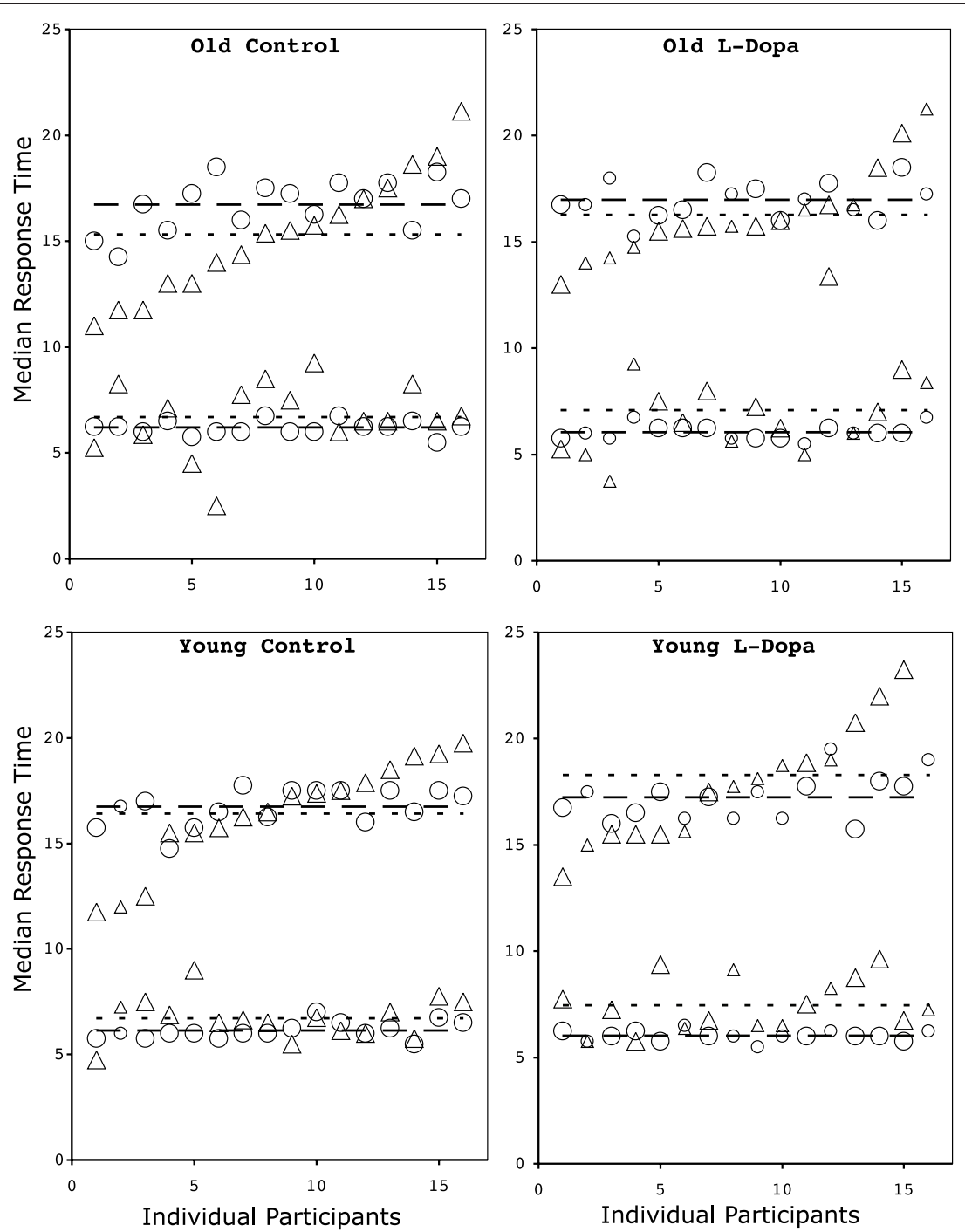

effect is mediated by the drug side effects, rather than drug action on the DA system.

Comparing the dashed lines in the two top panels to the dashed lines in the two bottom panels, one can see that the aged group's mean time productions during the retesting session were shorter than the young group's means, by about $0.83 \mathrm{sec}$, averaged over target interval and drug group, and subtracting the baseline data. This effect corresponds to a significant Session $\times$ Age group interaction $[F(1,60)=4.13, p<.05]$. Excluding the individuals that experienced nausea during retesting from the analysis increased the magnitude of the aging effect to $1.04 \mathrm{sec}$, and significance of the Session $\times$ Age group interaction $[F(1,52)=5.33, p<.05]$. The aged group's means for production of the two intervals were also closer together than those of the young group's by about $1.16 \mathrm{sec}$. However, the Session $\times$ Duration $\times$ Age group interaction was marginal $[F(1,60)=3.21, p=.078]$, until the individuals with nausea during testing were removed from the analysis $[F(1,52)=4.65, p<.05]$.
It is important to note that the Session $\times$ Age group $\times$ Drug group interaction was not significant either with $[F(1,60)=0.38]$ or without $[F(1,52)=0.55]$ those individuals who experienced nausea during the retesting session.

Table 1 presents the group mean time production NPCV (the nonparametric coefficient of variation) data by session and target interval duration. The NPCV measures the spread of responses relative to the median response time, and is equal for different target intervals when the scalar property applies. There were three significant effects in these data. The NPCVs of short interval productions were $1.3 \%$ greater than NPCVs of long interval production $[F(1,60)=22.28, p<.0001]$, a small but reliable violation of the scalar property. Retesting session NPCVs were $1.6 \%$ greater than baseline session NPCVs $[F(1,60)=26.21, p<.0001]$. There was also a significant Session $\times$ Duration $\times$ Drug group interaction $[F(1,60)=4.57, p<.05]$. This was a full crossover interaction, and the biggest increases in NPCV between 
Table 1. Time Production NPCV Data, with Standard Errors

\begin{tabular}{|c|c|c|c|c|c|}
\hline \multirow[b]{2}{*}{ Age Group } & \multirow[b]{2}{*}{ Drug Group } & \multicolumn{2}{|c|}{ Baseline Sessions } & \multicolumn{2}{|c|}{ Retesting Sessions } \\
\hline & & $6 \mathrm{sec}$ & $17 \mathrm{sec}$ & $6 \mathrm{sec}$ & $17 \mathrm{sec}$ \\
\hline Aged & Placebo & $9.02 \pm 1.03$ & $7.99 \pm 0.86$ & $9.80 \pm 1.02$ & $8.47 \pm 0.84$ \\
\hline Aged & Levodopa & $9.05 \pm 1.14$ & $6.78 \pm 0.83$ & $10.03 \pm 1.27$ & $8.89 \pm 1.61$ \\
\hline Young & Placebo & $7.16 \pm 0.93$ & $6.83 \pm 0.74$ & $10.72 \pm 1.22$ & $8.71 \pm 1.00$ \\
\hline Young & Levodopa & $6.76 \pm 0.78$ & $5.01 \pm 0.50$ & $7.98 \pm 0.70$ & $7.61 \pm 0.83$ \\
\hline
\end{tabular}

baseline and retesting sessions were in the two placebo groups' 6 -sec productions (2.2\%), and the two levodopa groups' 17 -sec productions (2.4\%). In contrast, increases in the two placebo groups' 17 -sec productions (1.2\%) and the two levodopa groups' 6 -sec productions (1.1\%) were smaller.

Removing participants who experienced nausea during the retesting session produced similar effects for duration $[F(1,52)=19.78, p<.0001]$, session $[F(1,52)=$ $24.14, p<.0001]$, and the Session $\times$ Duration $\times$ Drug group interaction $[F(1,52)=4.18, p<.05]$. Considering only the levodopa groups, the effects of session $[F(1,28)=18.78, p<.05]$ and duration $[F(1,28)=$ 17.16, $p<.05$ ] were significant, and the Session $\times$ Duration interaction was marginal $[F(1,28)=3.04$, $p=.092]$. All effects of the presence or absence of nausea were nonsignificant.

\section{Choice Reaction Time}

Individual participants' (symbols) and group mean (lines) $M D \mathrm{RT}$ data are presented in Figure 2. Aged participants were $206 \mathrm{msec}$ slower than young participants, a significant main effect of age $[F(1,60)=41.86$, $p<.0001]$. The Session $\times$ Age group interaction was significant $[F(1,60)=4.98, p<.05]$, reflecting a 55-msec improvement in the aged participants' speed between the baseline session and the retesting session that was absent in the young participants. The main effect of session $[F(1,60)=22.28, p<.05]$ was also significant. The absence of drug group effects renders side effects analyses unnecessary.

Table 2 presents the choice RT NPCV data. Aged participants' NPCVs were significantly higher than young participants' NPCVs $[F(1,60)=5.56, p<.05]$ by about $3.8 \%$. There was a tendency for the NPCVs to be lower in the retesting session than in the baseline session $[F(1,60)=$ $3.89, p<.0531]$. There were no significant effects on \%COR, and the cell means ranged from $96.6 \%$ to $98.3 \%$.

\section{Correlations between Time Production and Reaction Time Performance}

Table 3 presents the correlations between the difference in $M D$ RT between sessions and the difference in $M D$ time production between sessions. This table includes correlations among all participants (excluding those that felt nauseous during the retest session) as well as the various subgroupings of participants. None of the correlations is significant. Therefore, we cannot reject the null hypotheses that the difference in $M D$ RT between sessions is independent of the difference in either short or long interval $M D$ time production between sessions, for participants grouped by age, drug, or the interaction of the two.

\section{DISCUSSION}

In this experiment, a successful test of the hypothesis that an age-related time production deficit is DAmediated required three positive outcomes. First, we had to demonstrate that the age-related effect occurs in this procedure. That was accomplished by demonstrating a significant Age $\times$ Session and Age $\times$ Session $\times$ Duration interaction effect on the $M D$. Second, we had to demonstrate that levodopa had an effect on time production. The Session $\times$ Drug group interaction effect on the $M D$ demonstrated this effect. Third, we had to demonstrate an interaction of levodopa and aging effects, that is, a Session $\times$ Age group $\times$ Drug group interaction. However, that effect was not significant, and so we cannot reject the null hypothesis that the age-related time production effect observed in this experiment is independent of the alteration in DA function caused by a 200-mg dose of levodopa.

The successful demonstration of aging and levodopa effects in the current experiment (discussed in more detail below) created the necessary conditions for testing our main hypothesis, that the aging effect is DA-mediated. The absence of a significant interaction between the two effects instead supports the null hypothesis of independence between the effects. Failing to reject the null never rules out the possibility that the alternative is true. For example, a larger dose of levodo$\mathrm{pa}$, or another agent altogether, may result in a detectable interaction, where the current agent did not. We are especially interested in testing the effects of direct DA agonists, which may be more efficacious in reversing an age-related deficit stemming from reduced receptor density. Agents that may affect the many other neuro- 
Figure 2. Choice RT $M D$ data. The four panels each contain data for all 16 participants in one of the four groups, plus the group means. The two symbols in each column of each panel indicate one participant's mean $M D$ s for the baseline (circles) and retesting (triangles) sessions. The ordering of participants from left to right is the same as in Figure 1 . The solid lines are the group means for the baseline sessions and the dotted lines are the group means for the retesting sessions.

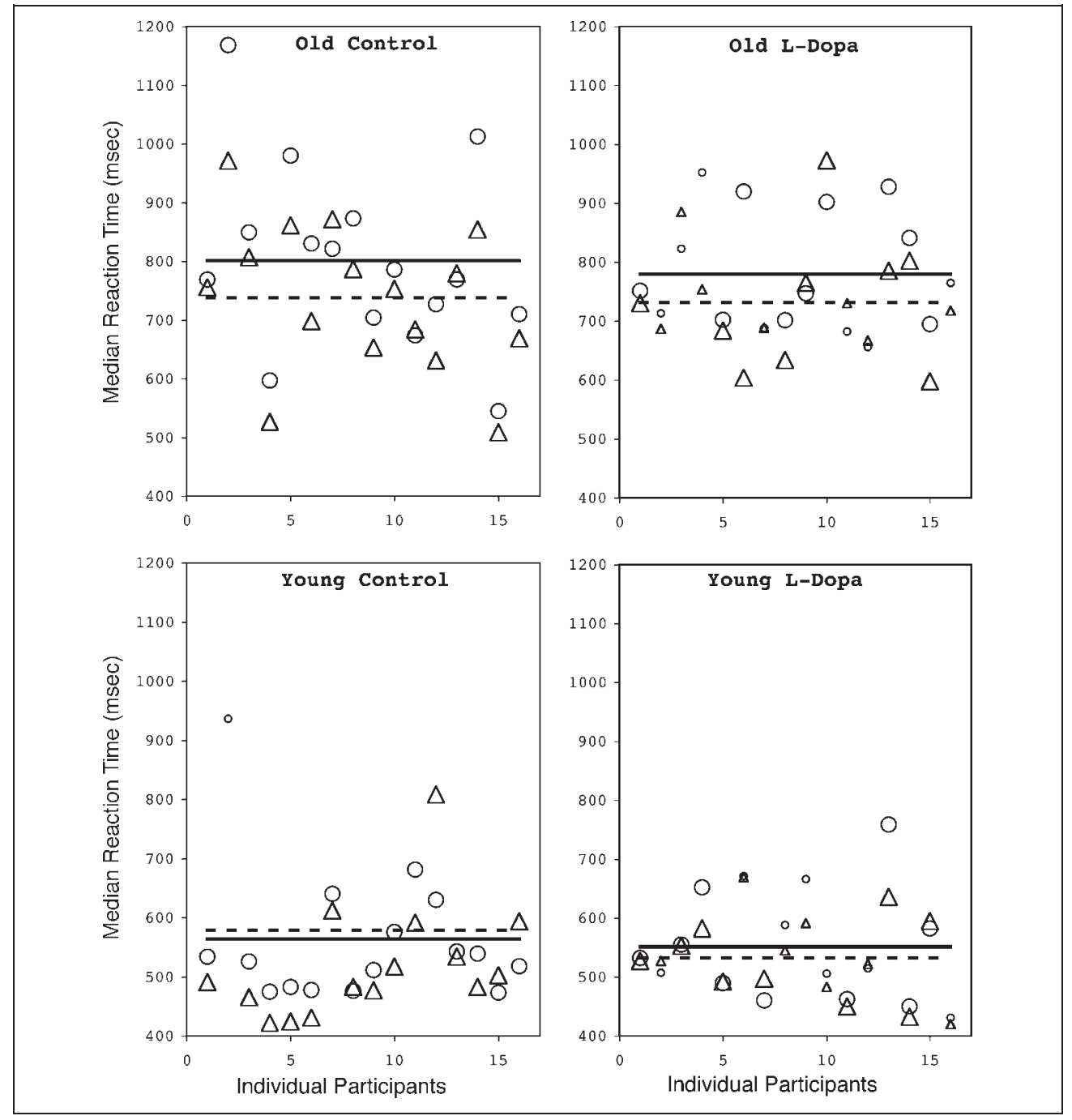

chemical systems shown to affect timing, including the cholinergic system (Meck, 2002; Meck \& Church, 1984), may also be good candidates. In addition, a failure to reject a null hypothesis may stem from a lack of task or design sensitivity. However, the current study task and design have now been employed to demonstrate the effects of levodopa on PD patients, as well as healthy young and old participants, and to demonstrate and replicate the basic effects of aging on time production, providing ample evidence of its sensitivity.

Table 2. Choice RT NPCV Data, with Standard Errors

\begin{tabular}{llcc}
\hline Age Group & Drug Group & Training & Testing \\
\hline Aged & Placebo & $22.74 \pm 5.92$ & $20.63 \pm 7.83$ \\
Aged & Levodopa & $22.92 \pm 6.59$ & $21.10 \pm 7.85$ \\
Young & Placebo & $20.97 \pm 13.11$ & $19.02 \pm 6.25$ \\
Young & Levodopa & $17.04 \pm 4.35$ & $15.34 \pm 3.42$ \\
\hline
\end{tabular}

The aging effect reported here replicates that observed in a previous study (Rakitin, Stern, et al., 2005) in two important ways. First, the effect occurs only during the delayed free-recall retesting session. Second, the direction of the production errors depended on the duration of the target time interval. That is, aged individuals overproduced the short interval and underproduced the long interval. In the previous study, the aging effect was somewhat smaller in magnitude on average (aged participants overproduced the short interval but accurately timed the long interval), but was more reliable. The difference between the studies may reflect random variability or differences in the procedures. The most obvious difference in the procedures is the duration of the retention interval between training and retesting - $1 \mathrm{hr}$ in the current study and $24 \mathrm{hr}$ in the previous study. A future study that parametrically varies the retention interval could resolve its effect on the magnitude and reliability of age-related time production errors.

The evidence for a levodopa-induced time production effect contrasts with the negative result obtained in the 
Table 3. Correlations between the Retest Session RT and Time Production Individual Medians Expressed as Difference from Baseline, Excluding Participants with Nausea during the Retesting Session

\begin{tabular}{lcccc}
\hline & & Old & Young & Old + Young \\
\hline Levodopa & $n$ & 12 & 13 & 25 \\
& Short & -.36176 & -.35262 & -.31474 \\
Control & Long & -.14978 & -.25756 & -.06191 \\
& $n$ & 16 & 15 & 31 \\
& Short & .11006 & -.05791 & .03787 \\
Levodopa + Control & $n$ & 28 & 28 & .30162 \\
& Long & .06445 & $.45519^{*}$ & 56 \\
& Short & -.17331 & -.15258 & -.13848 \\
& Long & -.03011 & .11582 & .12079 \\
\hline
\end{tabular}

Note: Rows labeled " $n$ " indicate the number of subjects included in that condition. Correlations (Pearson's $R$ ) in the rows labeled "Short" and "Long" are between the RT and time production of the short interval and long interval, respectively. None of these correlations is significant at the $p<.05$ level.

*Indicates $p<.1$ level.

only other study of the effects of levodopa on timing in healthy participants (Rammsayer, 1989). Three important differences between the current and previous study may account for the discrepancy. First, the previous study used a lower $(100 \mathrm{mg})$ dose of levodopa. ${ }^{3}$ There are no existing dose-response studies of the effects of levodopa on performance in healthy individuals, but two studies noting positive levodopa effects on RT used the current, higher (200 mg) dose (Hasbroucq et al., 2003; Micallef-Roll et al., 2001). Second, the current study tested much longer intervals than the previous study. There is psychophysical (Ivry \& Spencer, 2004; Gibbon et al., 1997), pharmacological (Rammsayer, 1994, 1997), and brain imaging (Lewis \& Miall, 2003) evidence to suggest that timing of shorter (i.e., $<1 \mathrm{sec}$ ) and longer intervals have different information processing requirements. Long interval timing puts greater demands on temporal memory and temporal attention. These processes are known to be DA mediated (e.g., Malapani, Deweer, et al., 2002; Meck \& Benson, 2002) and so the current experiment may have been more sensitive to DA effects. Third, this experiment required participants to retain temporal memories for at least an hour, whereas Rammsayer's study did not. This retention interval adds long-term (or reference) memory processing to the task, which may have further increased the task's sensitivity to DA.

The effect of levodopa in this experiment was to cause participants to respond too late relative to the target time interval. In terms of the standard information processing model of timing discussed in the Introduction, overproduction during testing implies that subjec- tive time is flowing slower in the retesting session (after drug administration) than during the baseline session, when the memory for the standard interval is established. One possibility is that this effect reflects psychomotor slowing not restricted to temporal cognition. However, this effect was specific to timing to the extent that levodopa did not produce lengthening of the RT in the choice RT task, nor was there an association between individuals' timing and RT performance. These two facts suggest that the effects of levodopa are specific to time production. However, this suggestion is tempered by the possibility that the null effect of levodopa on the RT reflects a lack of task sensitivity rather than an absence of an effect on psychomotor speed. Such a lack of sensitivity could explain our failure to replicate past positive findings (Hasbroucq et al., 2003; Micallef-Roll et al., 2001).

Several aspects of the internal clock model can account for the observed levodopa effect on time production. For example, if levodopa slows the pacemaker, inflates the remembered interval upon retrieval from long-term memory, or delays the closing of the switch when participants direct attention to time at the beginning of the trial, then participants will respond too late. It is possible to distinguish between some of these possibilities. Both pacemaker speed and memory translation effects can produce errors that are proportional to the duration of the target interval and preservation of the scalar property of timing variability (Malapani, Deweer, et al., 2002; Gibbon et al., 1984; Gibbon \& Church, 1983; Meck, 1983). In contrast, switch effects are associated with errors that are independent of the target interval duration (Meck \& Church, 1983) and may cause violations of the scalar property if the interval is sufficiently short (Rakitin, 2005). In the current data, the errors for the short interval and long interval are approximately equal, lending support to the delayed-switching hypothesis. The complex interaction evident in the variability data is not diagnostic of either effect, and is more likely indicative of baseline differences between the groups than the differential effects of levodopa. Taken together, the data favor the notion that application of levodopa slows the processes of switching attention to time.

Recent studies with rats of the effects of DA agonists on attention (Buhusi \& Meck, 2002) provide additional support for the delayed-switching hypothesis. When administered methamphetamine, rats tend to restart timing following an interruption in a timing stimulus. The DA antagonist haloperidol produced the opposite effect, and rats were able to readily switch attention between timing and not timing as the interruption began, and then switch back again when the gap ended. These results were interpreted to indicate that DA agonists could reduce attention to time-a finding consistent with the current results. In contrast, studies of pharmacological effects on pacemaker speed (Meck, 1983, 1986) indicate that DA agonists increase the speed 
of the internal pacemaker. Such results contradict the current data, which are consistent with a decrease in pacemaker speed after administration of levodopa.

Another important comparison is between the current results and the DA-mediated effects on temporal memory encoding observed in PD patients. PD patients trained off levodopa and then tested on medication (in an experiment very similar to the current one but with a 24-hr retention interval) overproduced two target intervals equal in duration to those used here (Malapani, Deweer, et al., 2002). The PD encoding effect is scalar in both magnitude and variability. Although errors of this sort can also be due to changes in pacemaker speed or long-term memory for duration, as discussed previously, the authors preferred to attribute this effect to a distortion in memory encoding. There were two reasons. First, it was assumed that the dysfunctional operation must have occurred during the baseline session when patients were in a DA-depleted state, and memory encoding is the most obvious candidate for producing an effect during a later retesting session when relearning of the interval was impossible. Second, the effect was in the opposite direction to that associated with DA pacemaker speed effects (Meck, 1983, 1986).

The similarities between the PD encoding effect and the current levodopa effect seem to end with the fact that both effects are characterized by overproduction of two intervals. Differences include the approximately constant errors in production of the two intervals and the association between the error and an increase in DA activity above baseline in the retesting session of this experiment. However, a few additional facts should be considered before entirely dismissing the comparison. First, medicated PD patients perform better than their age-matched controls (Malapani, Rakitin, et al., 2002), in terms of both accuracy and variability. This suggests that medicated PD patients may be demonstrating the effects of increased DA during the retesting session. Second, slowed pacemaker speed was considered as a possible mechanism in both cases, but was dismissed on the basis of indirect evidence. Third, discriminating between constant and proportional effects can be difficult when the effects are small, as was the case here. Considering these three facts together, it is conceivable that a relative increase in DA activity between training and testing, resulting in a slowed pacemaker during testing, could explain both effects if two additional facts were to come to light. First, some physiological mechanism for producing paradoxical DA agonist effects would have to be demonstrated. Some evidence for this already exists in the U-shaped dose-response curves sometimes associated with DA agonists (Misu et al., 1996; Arnsten et al., 1995), but these effects have not been shown in timing experiments aimed at understanding pacemaker speed. Second, future timing experiments with larger doses of levodopa or other DA agonists would have to demonstrate proportional timing errors with scalar variability.
Our tact in interpreting the results of the present experiment was to use SET to obtain as specific an inference as possible given the limitations of the experiment. However, these results can be interpreted more generally in two important ways. First, it is possible to conclude that levodopa affects attention to time without referring to a pacemaker-accumulator system. Several timing theories (e.g., Thomas \& Weaver, 1975; Wing \& Kristofferson, 1973) note that understanding the totality of timing psychophysics requires considering the relations between timing and nontiming processes in general. The Thomas and Weaver model, in particular, makes many of the same predictions as the switching model discussed here by assuming that attention is shared between visual and temporal processing, but does not make any reference to the switch.

Second, our expansive definition of the internal clock to include attention and memory components raises the question of the extent to which the phenomena reported in the present experiment are domain-specific timing effects. Our view is that in the absence of specific controlled experiments to test the hypothesis that temporal cognition shares information processing components with the processing of information from other domains, the data from the present experiments should be interpreted narrowly. However, as noted previously, both aging and dopaminergic drugs produce cognitive changes in a variety of domains. Thus, we must consider the possibility that the present effects are one instance of general aging and DA phenomenon made apparent by the complex nature of PI timing of relatively long intervals. More specifically, the suggestion that levodopa affects temporal attention by altering the operation of the switch component of the internal clock's time basis should not be taken to imply that the effects of levodopa on attention are specific to timing. On the contrary, that DA affects attention is a central component of a major theory of age-related cognitive decline (Braver \& Barch, 2002). The current experiment does not provide an opportunity to test the association between DA and attention in general because the attention demands of the current RT task are minimal. A future experiment on $\mathrm{DA}$ and timing that includes an RT task that requires sustained attention might prove useful in this regard.

\section{METHODS}

\section{Participants}

Thirty-two young participants were recruited from flyers posted on the campus of the Columbia University Medical Center. Thirty-two elderly participants were recruited from flyers posted on the campus of the Medical Center and through telephone contact using subject databases from previous studies. Interested participants were screened on the telephone or through e-mail for neurological or psychiatric disorders, use of 
psychoactive medication, and conditions that could potentially incur a health risk with the study drug, specifically glaucoma and melanoma. A neurologist at the testing site further screened the participants for medical history and current medical conditions. All participants assessed with the motor subscales of the Unified Parkinson's Disease Rating Scale (UPDRS; Richards et al., 1991) and were determined to be free of extrapyramidal signs (i.e., no subscale scores greater than 1). All participants were determined to be free of dementia, having obtained above 125 on the Mattis Dementia Rating Scale (DRS; Mattis, 1988) and above 45 on the modified Mini-Mental Status Exam (mMMSE; Stern, Sano, Paulson, \& Mayeux, 1987). Table 4 provides additional details about the participants.

The young and old participants were assigned randomly to receive either levodopa or a placebo control (see Procedures below). The final design therefore includes four groups of 16 participants: old/control, old/levodopa, young/control, and young/levodopa.

\section{Apparatus}

Data from all the participants were collected using a Macintosh G3 iBook laptop computer with a 13.1-in. color monitor. Participants were tested in rooms dedicated to running clinical experiments that were located in the Irving Center for Clinical Research at the Presbyterian Hospital of the Columbia University Medical Center. Stimulus presentation and response acquisition in the RT task were driven by the Psyscope experimental design package (Cohen, MacWhinney, Flatt, \& Provost, 1993), version 1.2.5. A custom-designed computer application was used to deliver the stimuli and record the responses for the time production task. Responses were recorded through the keyboard.

\section{Procedure}

All participants refrained from eating for a minimum of $2 \mathrm{hr}$ prior to arriving at the testing site at approximately

Table 4. Participant Demographics

\begin{tabular}{lll}
\hline & \multicolumn{1}{c}{ Aged } & \multicolumn{1}{c}{ Young } \\
\hline Age range & $60-86$ & $18-35$ \\
Age & $71.2 \pm 7.6$ & $24.4 \pm 4.4$ \\
\% Female & 53.125 & 53.125 \\
Education & $14.8 \pm 2.8$ & $15.3 \pm 2.1$ \\
UPDRS & $0.52 \pm 0.25$ & $0 \pm 0$ \\
mMMSE & $54.4 \pm 2.7$ & $55.4 \pm 1.9$ \\
DRS & $141.6 \pm 1.9$ & $142.9 \pm 1.2$ \\
\hline
\end{tabular}

Note: Values for age, education, UPDRS, mMMSE, and DRS are the mean \pm standard error. Education is in years. noon. Participants then provided informed consent and received onsite medical screening by a certified neurologist. They were then administered the neuropsychological tests, the choice RT task baseline session, and two time production task baseline sessions (one for each of two target intervals), in that order. After this, participants were administered either $200 \mathrm{mg}$ of levodopa with $50 \mathrm{mg}$ of carbidopa (i.e., Sinemet $100 / 25 \times 2$ ) or two tablets of a placebo, randomly determined, in a double-blind fashion. Following a 1-hr delay, participants were given the retesting sessions for the choice RT task and time production of the two target intervals.

The Columbia University Medical Center IRB approved all procedures.

\section{Tasks}

\section{Choice Reaction Time}

Choice RT task sessions consisted of 30 trials in one block. The baseline and retest sessions were identical. A warning signal ("+") appearing in the center of the screen indicated the beginning of a trial. Five hundred milliseconds after the warning signal appeared, it was replaced by a number ranging from 1 to 4 . Participants were instructed to press the " $\mathrm{z}$ " key with their left middle finger if the number is " 1 ," the " $x$ " key with their left index finger if the number is " 2 ," the "." key with their right index finger if the number is " 3 ," and the " " key with their right middle finger if the number is " 4 ." Participants were told to select their responses as quickly and accurately as possible. Feedback messages indicating if the response was "Correct" or "Incorrect" appeared $250 \mathrm{msec}$ after the clearing of stimulus and stayed on the screen for $1.25 \mathrm{sec}$. Alternatively, the feedback read "Sorry, ran out of time" if the participant failed to respond after $2000 \mathrm{msec}$.

\section{Time Production}

The time production task was a variant of the human PI task (Rakitin, Gibbon, et al., 1998) that included delayed free-recall retesting sessions without feedback (Rakitin, Stern, et al., 2005; Malapani, Deweer, et al., 2002). Production of the 6- and 17-sec target intervals occurred in separate blocks. The order in which the intervals were tested was counterbalanced across participants within group.

The baseline session for each interval consisted of 80 trials. The first 10 "fixed-time" trials demonstrated the target interval. On each trial, a blue square was presented until the target interval had elapsed, at which time it turned magenta for $1 \mathrm{sec}$ and then terminated. Participants were instructed to remember the duration of the blue square. The next 10 "peak-interval" (PI) trials began with the presentation of the blue square. Participants responded on each trial with a bout of at 
least four space bar presses that was supposed to be centered on the anticipated end of the target interval. The time of the first keypress is referred to as the "start" time and the time of the last keypress is referred to as the "stop" time. Following the response, the participants were shown feedback in the form of a graph indicating their response times relative to the target interval, and whether all responses were within $10 \%$ of the target interval. These trials were considered practice and were not included in the data analysis. The remaining 60 trials consisted of 30 PI trials with feedback, 15 PI without feedback, and 15 fixed-time trials.

The retest sessions for each interval consisted of 60 peak trials without feedback. The short and long intervals were tested in separate blocks and in the same order as the baseline session. There were no fixed-time trials (i.e., further demonstrations of the target intervals) or practice trials preceding either block. Before each block, participants were instructed to reproduce either the long or the short interval from the baseline session.

For the entire procedure, participants were instructed to refrain from counting or tapping. To further discourage counting, random digits were intermittently superimposed over the blue square. Interdigit times were determined randomly using two uniform distributions, 200-700 and 1300-1800 msec. All trials included the digit distracters.

\section{Analysis}

The mean of the start and stop times (i.e., the "middle") summarized time production performance for each PI trial (with or without feedback). The median $(M D)$ and the $\mathrm{NPCV}^{4}$ (i.e., the interquartile range/1.348\}/MD) of the trial middles summarized time production within each Subject $\times$ Duration $\times$ Session condition. The $M D$ was used to test hypotheses concerning production accuracy, or how close productions were to the target interval on average. The NPCV was used to test hypotheses concerning precision, or intraindividual variability, including the scalar property of timing variability, which predicts that target interval duration should not affect the NPCV. Hypotheses were tested using univariate ANOVA models. Each model included two betweenparticipant factors, age group and drug group, and two within-participant factors, target interval and session, in a full-factorial design. Models including the order of testing of the two target intervals (as a betweenparticipants factor) did not produce significant main or interaction effects, and this factor was dropped from the analyses.

The $M D$, NPCV, and percent correct (\%COR) summarized choice RT performance within each Subject $\times$ Session condition. Hypotheses were tested using univariate ANOVA models. Each model included two betweenparticipant factors, age group and drug group, and one within-participant factor, session, in a full-factorial design.
Two additional analyses were run for each task in order to examine the influence of side effects. One analysis used the standard model for each task, but excluded those participants who exhibited nausea during the retesting session. The other analysis subdivided the levodopa group participants into those that did or did not complain of nausea at any time after being given the drug. This analysis dropped drug group from the analysis and substituted nausea group as a betweenparticipants factor.

In order to explore further possible functional relationships between RT and time production task performance, we examined the correlations between RT and time production performance. Difference scores (baseline - retest) were computed from individuals' RT, short-interval time production, and long-interval time production medians. Correlations between the RT and the long-interval time production change scores and the RT and short-interval change scores were computed by age group, drug group, as well as the four groups of the Age group $\times$ Drug group interaction. Individuals expressing nausea during the retesting session were excluded from the analysis.

\section{Acknowledgments}

This research was supported in part by Columbia University's Parkinson's Disease Foundation, National Institute on Aging grant K01-AG000991 (awarded to B. C. R.), National Institute of Mental Health grant RO1-MH54793 (awarded to C. M.), and NIH grant MO1-RR00645 to Columbia University's General Clinical Research Center. We thank Kathy Kim and Tracy Sun for assistance with data collection, and Stephen Fairhurst for implementing custom software and data analysis methods. We also thank Richard B. Ivry and two anonymous reviewers for comments contributing to the improvement of this report.

Reprint requests should be sent to Brian C. Rakitin, $630 \mathrm{~W}$. 168th Street, P \& S Box 16, New York, NY 10032, or via e-mail: br130@columbia.edu.

\section{Notes}

1. The current use of the term "time production" is intended to distinguish tasks in which timing is assessed by measuring response latency from "time perception" tasks where participants make categorical timing responses, such as in the bisection procedure (e.g., Allan \& Gibbon, 1991). This definition differs from an older (e.g., Woodrow, 1951), more specific, and perhaps more common usage where time production refers to a task in which the participant generates a response with a latency matched to an interval stated in clock time (Allan, 1979). 2. The term "time basis" replaces the older term "clock stage." This substitution is intended to make a clear distinction between one stage of the information processing of intervals including the pacemaker, switch, and accumulator, and all cognitive processes necessary for interval timing referred to together as the "internal clock."

3. Rammsayer also used Madopar instead of Sinemet. The two drugs differ in the additive used to reduce associated nausea. Madopar adds benserazide hydrochloride, whereas Sinemet adds carbidopa. This difference is unlikely to have contributed to the studies' different outcomes. 
4. In order to avoid mixing parametric and nonparametric summary statistics, we generate a distribution relative scale parameter, the NPCV, by substituting the robust estimators of spread (the interquartile range) and central tendency (the $M D$ ) into the more familiar equation for the parametric scale estimator, the CV (or the standard deviation/the mean). In order to make the NPCV and the CV comparable in magnitude, the interquartile range has to be divided by 1.348 because the interquartile range accounts for a larger proportion of the normal distribution than does the standard deviation. That is, for a normal population, the standard deviation/interquartile range $=1.348$.

\section{REFERENCES}

Agid, Y., Cervera, P., Hirsch, E., Javoy-Agid, F., Lehericy, S., Raisman, R., \& Ruberg, M. (1989). Biochemistry of Parkinson's disease: 28 years later: A critical review. Movement Disorders, 4, S126-S144.

Allan, L. G. (1979). The perception of time. Perception $E$ Psychophysics, 26, 340-354.

Allan, L. G., \& Gibbon, J. (1991). Human bisection at the geometric mean. Learning and Motivation, 22, 39-58.

Arnsten, A. F., Cai, J. X., Steere, J. C., \& Goldman-Rakic, P. S. (1995). Dopamine D2 receptor mechanisms contribute to age-related cognitive decline: The effects of quinpirole on memory and motor performance in monkeys. Journal of Neuroscience, 15, 3429-3439.

Backman, L., Ginovar, N., Dixon, R. A., Wahlin, T. B. R., Wahlin, R., Wahlin, A., Halldin, C., \& Farde, L. (2000). Age-related cognitive deficits mediated by changes in the striatal dopamine system. American Journal of Psychiatry, 157, 635-637.

Block, R. A., Zakay, D., \& Hancock, P. A. (1998). Human aging and duration judgments: A meta-analytic review. Psychology and Aging, 13, 584-596.

Braver, T., \& Barch, D. M. (2002). A theory of cognitive control, aging cognition, and neuromodulation. Neuroscience and Behavioral Reviews, 26, 809-817.

Buhusi, C. V., \& Meck, W. H. (2002). Differential effects of methamphetamine and haloperidol on the control of an internal clock. Behavioral Neuroscience, 116, 291-297.

Cohen, J. D., MacWhinney, B., Flatt, M., \& Provost, J. (1993). Psyscope: A new graphic interactive environment for designing psychology experiments. Behavioral Research, Methods, and Computers, 25, 257-271.

Craik, F. I. M., \& Hay, J. F. (1999). Aging and judgments of duration: Effects of task complexity and method of estimation. Perception \& Psychophysics, 61, 549-560.

Diedrichsen, J., Ivry, R. B., \& Pressing, J. (2003). Cerebellar and basal ganglia contributions to interval timing. In W. H. Meck (Ed.), Functional and neural mechanisms of interval timing (pp. 457-484). Boca Raton, FL: CRC Press.

Frederick, D. L., \& Allen, J. D. (1996). Effect of selective dopamine $\mathrm{D}^{1}$ and $\mathrm{D}^{2}$-agonists and antagonists on timing performance in rats. Pharmacology, Biochemistry and Behavior, 53, 759-764.

Gibbon, J., \& Church, R. M. (1983). Sources of variance in an information processing theory of timing. In H. L. Roitblat, T. G. Bever, \& H. S. Terrace (Eds.), Animal cognition (pp. 465-488). Hillsdale, NJ: Erlbaum.

Gibbon, J., Church, R. M., \& Meck, W. (1984). Scalar timing in memory. Annals of the New York Academy of Sciences, 423, 52-77.

Gibbon, J., Malapani, C., Dale, C., \& Gallistel, C. R. (1997). Toward a neurobiology of temporal cognition: Advances and challenges. Current Opinion in Neurobiology, 7, 170-179.
Hasbroucq, T., Tandonnet, C., Micallef-Roll, J., Blin, O., \& Possamaï, C.-A. (2003). An electromyographic analysis of the effect of levodopa on the response time of healthy subjects. Psychopharmacology, 165, 313-316.

Haycock, J. W., Becker, L., Ang, L., Furukawa, Y., Hornykiewicz, O., \& Kish, S. J. (2003). Marked disparity between agerelated changes in dopamine and other presynaptic dopaminergic markers in human striatum. Journal of Neurochemistry, 87, 574-585.

Hornykiewicz, O. (1989). Ageing and neurotoxins as causative factors in idiopathic Parkinson's disease-A critical analysis of the neurochemical evidence. Progress in Neuro-Psychopharmacology and Biological Psychiatry, 13, 319-328.

Ivry, R. B., \& Hazeltine, E. (1995). Perception and production of temporal intervals across a range of durations: Evidence for a common timing mechanism. Journal of Experimental Psychology: Human Perception \& Performance, 21, 3-18.

Ivry, R. B., \& Spencer, R. M. C. (2004). The neural representation of time. Current Opinion in Neurobiology, 14, 225-232.

Kaasinen, V., \& Rinne, J. O. (2002). Functional imaging studies of dopamine system and cognition in normal aging and Parkinson's disease. Neuroscience and Biobehavioral Reviews, 26, 785-793.

Kaasinen, V., Vilkman, H., Hietala, J., Nagren, K., Helenius, H., Olsson, H., Farde, L., \& Rinne, J. O. (2000). Age-related dopamine, $\mathrm{D}^{2} / \mathrm{D}^{3}$ receptor loss in extrastriatal regions of the human brain. Neurobiology of Aging, 21, 683-688.

Lewis, P. A., \& Miall, R. C. (2003). Distinct systems for automatic and cognitively controlled time measurement: Evidence from neuroimaging. Current Opinion in Neurobiology, 13, 250-255.

Lustig, C., \& Meck, W. (2001). Paying attention to time as one gets older. Psychological Science, 12, 478-484.

Malapani, C., Deweer, B., \& Gibbon, J. (2002). Separating storage from retrieval dysfunction of temporal memory in Parkinson's disease. Journal of Cognitive Neuroscience, 14, 311-322.

Malapani, C., \& Rakitin, B. C. (2003). Interval timing in the dopamine-depleted basal ganglia: From empirical data to timing theory. In W. H. Meck (Ed.), Functional and neural mechanisms of interval timing (pp. 485-514). Boca Raton, FL: CRC Press.

Malapani, C., Rakitin, B. C., Fairhurst, S., \& Gibbon, J. (2002). Neurobiology of timing. Cognitive Processing, 3, 3-20.

Malapani, C., Rakitin, B. C., Levy, R., Meck, W. H., Deweer, B., Dubois, B., \& Gibbon, J. (1998). Coupled temporal memories in Parkinson's disease: A dopamine-related dysfunction. Journal of Cognitive Neuroscience, 10, 316-331.

Maricq, A. V., \& Church, R. M. (1983). The differential effects of haloperidol and methamphetamine on time estimation in the rat. Psychopharmacology, 79, 10-15.

Maricq, A. V., Roberts, S., \& Church, R. M. (1981). Methamphetamine and time estimation. Journal of Experimental Psychology: Animal Behavior Processes, 7, 18-30.

Mattis, S. (1988). Dementia Rating Scale (DRS). Odessa, FL: Psychological Assessment Resources.

Mattle, M, \& Meck, W. H. (2000). Neurophysiological mechanisms of interval timing behavior. BioEssays, 22 , 93-103.

McCormack, T., Brown, G., Maylor, E., Darby, R., \& Green, D. (1999). Developmental changes in time estimation: Comparing childhood and old age. Developmental Psychology, 35, 1143-1155. 
Meck, W. H. (1983). Selective adjustment of the speed of the internal clock and memory processes. Journal of Experimental Psychology: Animal Behavior Processes, 9, 171-201.

Meck, W. H. (1986). Affinity for the dopamine $\mathrm{D}^{2}$ receptor predicts neuroleptic potency in decreasing the speed of an internal clock. Pharmacology, Biochemistry, and Behavior, 25, $1185-1189$.

Meck, W. H. (1996). Neuropharmacology of timing and time perception. Cognitive Brain Research, 3, 227-242.

Meck, W. H. (2002). Choline uptake in the frontal cortex is proportional to the absolute error of a temporal memory translation constant in mature and aged rats. Learning and Motivation, 33, 88-104.

Meck, W. H., \& Benson, A. M. (2002). Dissecting the brain's internal clock: How frontal-striatal circuitry keeps time and shifts attention. Brain and Cognition, 48, 195-211.

Meck, W. H., \& Church, R. M. (1983). A mode control model of counting and timing processes. Journal of Experimental Psychology: Animal Behavior Processes, 9, 320-334.

Meck, W. H., \& Church, R. M. (1984). Cholinergic modulation of the content of temporal memory. Behavioral Neuroscience, 101, 457-464.

Micallef-Roll, J., Rihet, P., Hasbroucq, T., Possamai, C., \& Blin, O. (2001). Levodopa-induced drowsiness in healthy volunteers: Results of a choice reaction time test combined with a subjective evaluation of sedation. Clinical Neuropharmacology, 24, 91-94.

Misu, Y., Goshima, Y., Ueda, H., \& Okamura, H. (1996). Neurobiology of L-dopaergic systems. Progress in Neurobiology, 49, 415-454.

Newman, R. P., LeWitt, P. A., Jaffe, M., Calne, D. B., \& Larsen, T. A. (1985). Motor function in the normal aging population: Treatment with levodopa. Neurology, 35, 571-573.

O'Boyle, D. J., Freeman, J. S., \& Cody, F. W. J. (1996). The accuracy and precision of timing of self-paced, repetitive movements in subjects with Parkinson's disease. Brain, $119,51-70$

Ollat, H. (1992). Dopaminergic insufficiency reflecting cerebral ageing: Value of a dopaminergic agonist, piribedil. Journal of Neurology, 239, S13-S16.

Pastor, M. A., Artieda, J., Jahanshahi, M., \& Obeso, J. A. (1992). Time estimation and reproduction is abnormal in Parkinson's disease. Brain, 115, 211-225.

Pastor, M. A., Jahanshahi, M., Artieda, J., \& Obeso, J. A. (1992). Performance of repetitive wrist movements in Parkinson's disease. Brain, 115, 875-891.

Perbal, S., Droit-Volet, S., Isingrini, M., \& Pouthas, V. (2002). Relationships between age-related changes in time estimation and age-related changes in processing speed, attention, and memory. Aging, Neuropsychology and Cognition, 9, 201-216.

Rakitin, B. C. (2005). Effects of spatial stimulus-response compatibility on choice time production accuracy and variability. Journal of Experimental Psychology: Human Perception and Performance, 31, 685-702.

Rakitin, B. C., Gibbon, J., Penney, T. B., Malapani, C., Hinton, S. C., \& Meck, W. H. (1998). Peak-interval timing in humans. Journal of Experimental Psychology: Animal Behavior Processes, 24, 19-33.

Rakitin, B. C., Stern, Y., \& Malapani, C. (2005). The effects of aging on time production in delayed free-recall. Brain and Cognition, 58, 17-34.

Rammsayer, T. (1989). Is there a common dopaminergic basis of time perception and reaction time? Neuropsychobiology, 21, 37-42.

Rammsayer, T. H. (1994). A cognitive-neuroscience approach for elucidation of mechanisms underlying temporal information processing. International Journal of Neuroscience, 77, 61-76.

Rammsayer, T. H. (1997). Are there dissociable roles of the mesostriatal and mesolimboccortical dopamine systems on temporal information processing in humans? Neuropsychobiology, 35, 36-45.

Rammsayer, T. H. (1999). Neuropharmacological evidence for different timing mechanisms in humans. Quarterly Journal of Experimental Psychology, 52B, 273-286.

Rammsayer, T. H., \& Vogel, W. H. (1992). Pharmacologic properties of the internal clock underlying time perception in humans. Neuropsychobiology, 26, 71-80.

Richards, M., Marder, K., Bell, K., Dooneief, G., Mayeux, R., \& Stern, Y. (1991). Interrater reliability of extrapyramidal signs in a group assessed for dementia. Archives of Neurology, 48, $1147-1149$.

Santi, A., Weise, L., \& Kuiper, D. (1995). Amphetamine and memory for event duration in rats and pigeons: Disruption of attention to temporal samples rather than changes in the speed of the internal clock. Psychobiology, 23, 224-232.

Shea-Brown, E. T., Rinzel, J., Rakitin, B. C., \& Malapani, C. (in press). A firing-rate model of Parkinson's disease deficits in interval timing. Cognitive Brain Research.

Stanford, L., \& Santi, A. (1998). The dopamine D ${ }^{2}$ agonist quinpirole disrupts attention to temporal signals without selectively altering the speed of the internal clock. Psychobiology, 26, 258-266.

Stern, Y., Sano, M., Paulson, J., \& Mayeux, R. (1987). Modified Mini-Mental State Examination: Validity and reliability. Neurology, 37, 179.

Suhara, T., Fukuda, H., Inoue, O., Itoh, T., Suzuki, K., Yamasaki, T., \& Tateno, Y. (1991). Age-related changes in human $\mathrm{D}^{1}$ receptors measured by positron emission tomography. Psychopharmacology, 103, 41-45.

Thomas, E. A. C., \& Weaver, W. B. (1975). Cognitive processing and time perception. Perception \& Psychophysics, 17, 363-367.

Treisman, M. (1963). Temporal discrimination and the indifference interval: Implications for a model of the "internal clock." Psychological Monographs, 77, 1-13.

Vanneste, S., \& Pouthas, V. (1999). Timing in aging: The role of attention. Experimental Aging Research, 25, 49-67.

Volkow, N. D., Ruben, G. C., Wang, G.-J., Fowler, J. S., Moberg, P. J., Ding, Y.-S., Hitzemann, R., Smith, G., \& Logan, J. (1998). Association between decline in brain dopamine activity with age and cognitive and motor impairment in healthy individuals. American Journal of Psychiatry, 155, 344-349.

Volkow, N. D., Wang, G. J., Fowler, J. S., Logan, J., Gatley, S. J., Mac Gregor, R. R., Schlyer, D., Hitzemann, R., \& Wolf, A. (1996). Measuring age-related changes in dopamine $\mathrm{D}^{1} / \mathrm{D}^{2}$ receptors with ${ }^{11} \mathrm{C}$-raclopride and ${ }^{18} \mathrm{~F}-\mathrm{N}$-methylspiroperidol Psychiatry Research, 31, 11-16.

Wearden, J. H., \& McShane, B. (1988). Interval production as an analogue of the peak procedure: Evidence for similarity of human and animal timing processes. Quarterly Journal of Experimental Psychology, 40B, 363-375.

Wearden, J., Wearden A., \& Rabbitt, P. (1997). Age and IQ effects on stimulus and response timing. Journal of Experimental Psychology: Human Perception and Performance, 23, 962-979.

Wing, A. M., \& Kristofferson, A. B. (1973). Response delays and the timing of discrete motor responses. Perception $\mathcal{E}$ Psychophysics, 14, 5-12.

Woodrow, H. (1951). Time perception. In S. S. Stevens (Ed.), Handbook of experimental psychology (pp. 1224-1236). New York: Wiley.

Zakay, D., \& Block, R. A. (1997). Temporal cognition. Current Directions in Psychological Science, 6, 12-16. 